\title{
Characterization of Pen n 13, a Major Allergen from the Mold Penicillium notatum
}

\author{
Lu-Ping Chow,* Shyh-Horng Chiou,† Meng-Chin Hsiao,* Chia-J ung Yu,* and Bor-Luen Chiang§ \\ *Institute of Biochemistry, College of Medicine and §Graduate Institute of Clinical Medicine, College of Medicine, \\ National Taiwan University, Taiped, Taiwan 100, Republic of China; and †Institute of Biological Chemistry, \\ Academia, Taipei, Taiwan, Republic of China
}

Penicillium notatum is a well-known indoor aeroallergen and is frequently included in skin test panels for allergic diagnosis. On two-dimensional immunoblotting using patients' sera containing I gE and monoclonal antibody D7B8 specific for Pen C 1 of P. citrinum, two allergens with a molecular mass of $33 \mathrm{kDa}$ but different isoelectric points were identified. A novel cDNA coding for Pen $\mathbf{n} \mathbf{1 3}$ was cloned and sequenced. The nucleotide sequence codes for a protein 397 amino acids including a putative signal peptide of 25 amino acids and a propeptide of 90 amino acids. The allergen is an alkaline serine protease that shares more than $39 \%$ identical residues with other kinds of mold allergens. The coding CDNA of Pen $\mathbf{n} 13$ was cloned into vector PQE-30 and expressed in E. coli M15 as a His-tag fusion protein and purified to homogeneity. The fusion protein reacted with monoclonal antibodies of Pen C 1 and with IgE from Penicillium-allergic patients. Furthermore, it also cross-reacted strongly with IgE specific for the natural Pen c 1, indicating that similar IgE binding epitopes may exist in the allergens of P. notatum and P. citrinum. Antigenicity index plots indicated that there are several similar epitope regions of high antigenic indices in Pen c 1 and Pen $n$ 13, corroborating that mold allergens belonging to the alkaline serine protease family possess similar protein structure and strong antigenic cross-reactivity. $\odot 2000$ Academic Press

Key Words: mold allergens; IgE-binding activity; alkaline serine protease; Penicillium notatum.

Atopic disorders, such as asthma, are increasingly prevalent in the developed countries. Fungal spores are universal atmospheric components and were long recognized as important causes of respiratory allergy (1). Over

Abbreviations used: RACE, rapid amplification of CDNA ends; TBS, Tris-buffered saline; IPTG, isopropyl-1-thio- $\beta$-D-galactopyranoside.
60 species of fungi are known to be allergenic to human. Penicillium notatum is a well-known indoor mold and is frequently included in skin test panels for allergic diagnosis (2). Several allergens produced by mold have been doned and their biological function and mechanism for eliciting allergic reactions are not completely understood in detail (2). More recently, molecular characterization of allergens from the mold $\mathrm{P}$. citrinum has been performed, and the complete cDNAs encoding two allergens of the major allergens Pen c 1 and Pen c 2, have been cloned and expressed $(3,4)$. So far, only one 68-kDa major glycoprotein allergen with IgE binding capacity from $P$. notatum was doned and shown to share sequence homology with $\beta$-N-acetyl gl ucosaminidase (5).

The cross-reactivity of molds have been investigated in several reports. Notably the allergenic crossreactivity among the 33-kDa major allergens of $\mathrm{P}$. citrinum, $\mathrm{P}$. notatum and $\mathrm{P}$. brevicompactum was detected previously (6). In a recent report, IgE crossreactivity among proteases-type allergens of A. flavus and $P$. citrinum has also been demonstrated (7). These studies revealed the presence of shared allergenic activity among different mold species.

In a previous study of $P$. notatum, a protein of $34 \mathrm{kDa}$ was found to react with more than $24 \%$ of 70 asthmatic sera (8). The allergen from Penicillium notatum deserves to be thoroughly investigated due to its high prevalence and unique allergenic property. This novel allergen from P. notatum was identified by two-dimensional immunoblotting and monoclonal antibodies (mAbs), and subsequently its partial sequence was determined by $\mathrm{N}$-terminal microsequencing. These studies were herein followed by the molecular cloning and expression of this allergen in Escherichia coli. The cloned allergen was thus designated Pen $\mathrm{n} 13$ according to the recommendation of International Union of Immunological Societies (9). Pen n 13-encoding cDNA was subd oned into the plasmid pQE 30 and expressed in E. coli. The recombinant protein was purified and characterized with regard to their immuno- 
logical cross-reactivities with two monoclonal antibodies and patients' IgE.

\section{MATERIALS AND METHODS}

Patients' sera and monoclonal antibodies. Sera were from three Penicillium-sensitive patients who confirmed by clinical history and characterized by CAP measurements (CAP score 3 or higher) for IgE reactivity (Pharmacia Diagnostics $A B$, U ppsala, Sweden). Sera from non-allergic individuals were collected and used as a negative control. One mAb, D7B8 directed against purified Pen $c 1$ was used for the characterization of mold allergen. Another mAb 55A against the Pen C 1 of P. citrinum was used previously (10).

Allergen extraction and two-dimensional PAGE. P. notatum was obtained from the F ood I ndustry Research \& Development Institute, Hsinchu, Taiwan (CCRC 30568). Fungal matts of P. notatum containing both spores and mycelia were collected from CzapekDox broth medium (Difco, Detroit, MI). About one gram of Iyophilized matts was ground in a mortar with liquid nitrogen, ground mycelia and spores were then mixed with phosphate-buffered saline extraction buffer at $4^{\circ} \mathrm{C}$ for $1 \mathrm{~h}$ with constant stirring. After centrifugation, the supernatant was precipitated with trichloroacetic acid to a final concentration of $5 \%$. The resultant precipitate was stored at $-70^{\circ} \mathrm{C}$. Dried powder ( $2 \mathrm{mg}$ ) was dissolved in the isoelectric focusing sample buffer (9.0 M urea, 2\% pharmalyte, $60 \mathrm{mM}$ DTT, 0.5\% Triton X-100 and $0.003 \%$ bromophenol blue) and two-dimensional PAGE was carried out by a procedure described previously (11). In the first dimension IEF, an immobilized $\mathrm{pH}$ gradient of 3 to 10 was used on a horizontal electrophoresis system, Multiphor II (Pharmacia Co., Sweden); for the second dimension, SDS-PAGE was performed according to Laemmli (12).

SDS-PAGE and immunoblotting. Proteins from mold extracts were separated by SDS-PAGE in $12.5 \%$ polyacrylamide gel under reducing conditions. After electrophoresis, the separated proteins were electrophorectically transferred to PVDF membranes. In experiments of IgE binding assay, the blots were blocked with $1 \%$ skimmed milk in Tris-buffered saline (TBS) for $1 \mathrm{~h}$ at room temperature. After washing with TBS, blots were incubated with diluted patients' pooled sera at $4^{\circ} \mathrm{C}$ for $16 \mathrm{~h}$. After washing, the membrane blots were incubated with alkaline phosphatase-conjugated monoclonal anti-human IgE antibodies (Pharmingen, San Diego, CA) at room temperature for $1 \mathrm{~h}$. Bound antibodies were detected with a BCIP/ NBT system (3). To detect mAbs binding reactivity, horseradish peroxidase-conjugated goat anti-mouse IgG was used as secondary antibody using a substrate solution of acetate buffer containing 3-amino-9-ethyl-carbazole and hydrogen peroxide (3).

$\mathrm{N}$-terminal microsequencing. N-terminal Edman degradation of the allergen Pen $n 13$ was performed on a PROCISE 494 protein sequencer (Perkin EImer/ABI, Foster City, CA) equipped with an on-line detection of phenylthiohydantoin derivatives.

Preparation of mRNA from P. notatum, PCR amplification and cloning. mRNA was extracted from mycelia according to the method of Chomczynski and Sacchi (13). Poly(A) ${ }^{+}$RNA was purified with the oligo(dT) cellulose kit (Pharmacia, Uppsala, Sweden). One $\mu \mathrm{g}$ of mRNA was reverse transcribed using the Marathon amplification kit (Clontech Laboratories, Palo Alto, CA), and the doublestrand cDNAs were ligated overnight by T4 DNA ligase with the adaptor provided by the kit. Two primers were designed based on the $\mathrm{N}$-terminus and $3^{\prime}$-coding region of $\mathrm{Pen} \mathrm{C} 1$ from $\mathrm{P}$. citrinum (3), for 5' primer (5'-ATGGGTTTCCTCAAGGTCCTC-3') and 3' primer (5'TTAGACGTTGATGCCATTGTA-3' with the stop codon). Coding sequence of the Pen $n 13$ gene was thus amplified by PCR. The amplified products were analyzed by electrophoresis and subcloned into pGEM-T vector (Promega, Madison, WI) and then used to transform E. coli J M 109. DNA sequencing was performed using the PRISM dye terminator cycle sequencing kit and a 373-A DNA sequencer (Applied Biosystems, Foster City, CA).

Production and purification of recombinant Pen n 13 (rPen n 13). To express the mature allergen Pen $n 13$ in E. coli, the cDNA was modified by PCR to remove the signal and pro-peptides sequences. Specific primers were designed to include a BamHI site at the $5^{\prime}$ end and a stop codon at the $3^{\prime}$ end of the cloned CDNA. The primers used were 5'-GCGGATCCGCGAACGTGGTTCAATCCAAT-3' (sense primer) and 5'-TTAGACGTTGATGCCATTGTATAG-3' (antisense primer). By ligating the coding sequences into the BamHI-Pstl restriction sites of the pQE-30 expression vector, Pen $n 13$ was expressed as a hexahistidine-tagged fusion protein in transformed E. coli M15 cells. (QIAGEN, Chatsworth, CA). The culture was induced by IPTG until the the $A_{600} \mathrm{~nm}$ of the medium reached 0.6. The culture was further incubated $2 \mathrm{~h}$. Then the product was purified under denatured condition by $\mathrm{Ni}$-chelate affinity chromatography according to the protocol. I mmunological detection was performed with serum pool from Penicillium sensitized patients and mAbs D7B8 and 55A.

Cross-inhibition experiments. Native Pen c 1 was isolated from $P$. citrinum culture according to the previous study (3). For immunobl ot inhibition assays, a serum pool of mold-allergic patients was incubated with purified rPen $\mathrm{n} 13(20 \mu \mathrm{g} / \mathrm{ml})$, BSA $(20 \mu \mathrm{g} / \mathrm{ml})$, and buffer as control for $2 \mathrm{~h}$ at $4^{\circ} \mathrm{C}$. Subsequently, the remaining IgE-binding capacity was measured accordingly (7).

\section{RESULTS AND DISCUSSIONS}

\section{Allergen Identification and Microsequencing}

In order to localize and identify the cross-reactive mold allergens, the two-dimensional PAGE blots were incubated with pooled human sera and the Pen $\mathrm{C}$ 1-specific mAb D7B8. Protein staining of the twodimensional PAGE blot with Coomassie brilliant blue (Fig. 1A) revealed more than 150 protein spots. To identify spots corresponding to the allergens, IgEbinding spots in two-dimensional PAGE were visualized by immunoblotting using sera from Penicilliumsensitized patients. After immunoblotting, six different spots with molecular masses of 28 to $66 \mathrm{kDa}$ and $\mathrm{pl}$ values ranging from about 4.5 to greater than 6.5 were shown to be reactive to IgE antibodies (Fig. 1B). To identify the IgE binding protein in P. notatum with possible cross-reactivity to Pen c 1 , immunoreactive protein spots were characterized again with $\mathrm{MAb}$ D7B8 directed against Pen $\mathrm{c} 1$, the allergen from $P$. citrinum (Fig. 1C). For negative control, no positive spots were detected when the normal serum was used (data not shown). The protein spots $A$ and $B$ indicated by the arrows in Fig. $1 \mathrm{~A}-1 \mathrm{C}$ show the allergens with immunological cross-reactivity to Pen c 1 . They are proteins of same apparent molecular weight and different isoelectric values ( $\mathrm{pl}$ ). Protein spot $\mathrm{A}$ has an $\mathrm{N}$-terminal sequence of ANVVQSNVPSWGL, which is identical to that of the Pen $\mathrm{C} l$ allergen of $\mathrm{P}$. citrinum.

\section{Sequence Analysis and Derived Amino Acid Sequence of the Pen $n 13$ cDNA}

The sequence of the CDNA clone encoding Pen $n 1$ is shown in Fig. 2. The Pen n 13 cDNA contains an open 
A

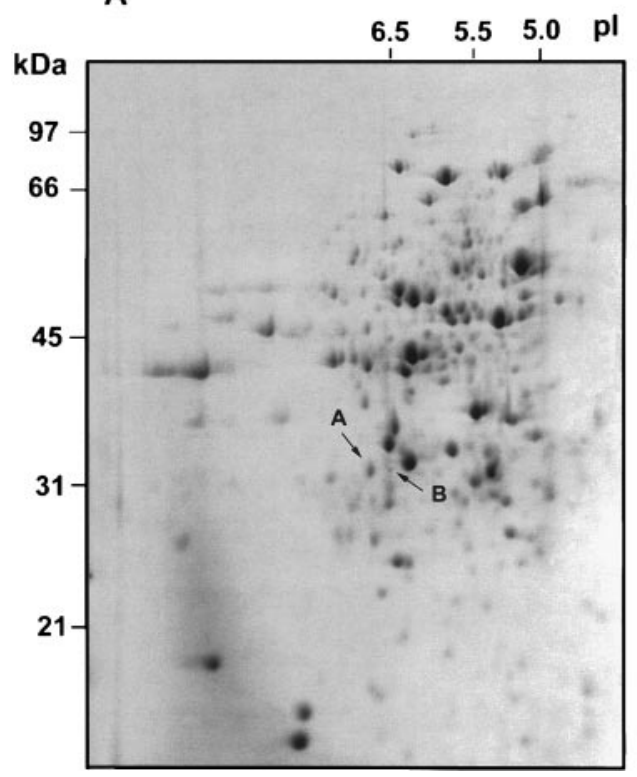

B

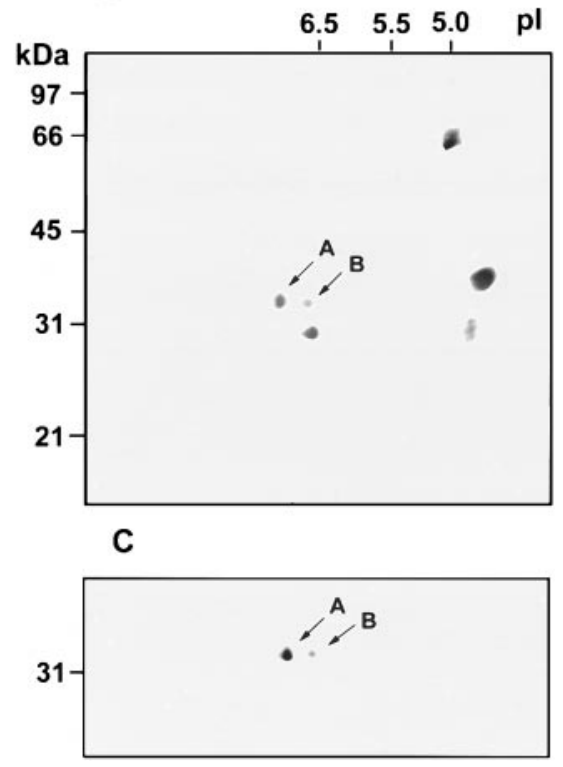

FIG. 1. Two-dimensional immunoblot analysis. (A) Two-dimensional blot of P. notatum crude extract was visualized with Coomassie brilliant blue staining. (B) Two-dimensional blot of A was immunoblotted with the reactive patients' pooled sera. (C) Two-dimensional blot of $A$ was immunoblotted with the monoclonal D7B8 against Pen $c 1$. The arrows $A$ and $B$ indicate the immunoreactive spots to serum IgE antibodies and $\mathrm{mAbs}$, which correspond to protein spots $\mathrm{A}$ and $\mathrm{B}$ of allergen proteins in $\mathrm{A}$. Protein spot $\mathrm{A}$ correspond to the allergen Pen $\mathrm{n}$ 13 as identified by $\mathrm{N}$-terminal microsequencing.

reading frame of 1194 nucleotides corresponding to a polypeptide of 397 amino acids. The 25 amino acid segment at the beginning of the deduced amino acid sequence appear to be a signal peptide since it consists of a long stretch of hydrophobic amino acid. The predicted signal peptide is followed by 90 amino acids of pro-sequence which precedes the $\mathrm{N}$-terminal amino acid segment of the native protein. Therefore, Pen $n 13$ is probably synthesized as a prepro-enzyme with a rather long propeptide containing a 90-amino-acid segment that is not part of the native form. Most proteases in the serine protease family possess a precursor structure with a long propeptide (14). The biological function of the propeptide is thought to prevent the protease from autolysis until it is processed (15).

\section{Comparison of Pen $\mathrm{n} 13$ Protein Sequences with Other Related Allergens}

Sequence similarities between the deduced amino acid sequence of Pen $\mathrm{n} 13$ and other mold allergens were also examined (Fig. 3). Sequence analysis revealed that Pen $n 13$ showed $99 \%, 41 \%$ and $39 \%$ sequence identity to Pen c 1 (3), Asp f $13(16,17)$, and Asp fl 1 (7), respectively. Based on sequence similarity, we could deduce that catalytic amino acid residues probably locate at Asp 42, His 73 and Ser 228, which are also commonly found for various members of serine protease family (18). One possible disulphide bridges ap- pears to be Cys182-Cys253. Three peptide segments at the 34th to 36th, 69th to 71st and 72nd to 74th amino acid residues with distinct $\mathrm{N}-\mathrm{X}-\mathrm{S}$ sequence feature are potential glycosylation sites. The extensive similarities (99\%) between the allergens from P. citrinum and P. notatum is indicative of the close relatedness of these two mold species.

\section{Expression and Purification of Recombinant Allergen rPen n 13}

cDNA encoding allergen Pen $n 13$ was expressed as a hexahistidine-tagged fusion protein in E. col i M15. The heterologous expression of the mold allergen resulted in generation of a prominent protein band with an apparent molecular mass of $36 \mathrm{kDa}$ in a much higher yield then than other expressed proteins of $E$. coli cells (Fig. 4A). Further purification by $\mathrm{Ni}$-chelate chromatography led to a homologous protein fraction of $r P e n n$ 13 (lane 3 of Fig. 4A).

Purified $r$ Pen $n 13$ was test in immunoblots with pool sera of patients and anti-Pen c 1 mAbs D7B8 and 55A (Fig. 4B). rPen $\mathrm{n} 13$ showed strong reaction to sera I gE from patients with allergy, indicating that the allergenic properties are well preserved in the recombinant derived protein. None of the normal sera showed any reactivity with this protein (data not shown). Both mAbs D7B8 and 55A, raised against native Pen c 1 , displayed binding reactivity to rPen $n$ 13, indicting 


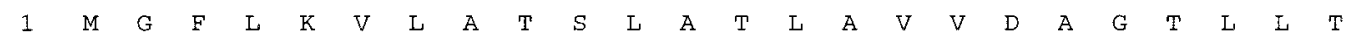
ATG GGT TTC CTC AAG GTC CTC GCT ACA TCT CTT GCG ACT CTT GCA GTC GTC GAT GCT GGT ACT CTC CTT ACC 72

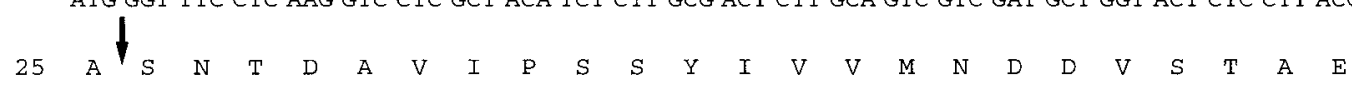
GCC AGC AAC ACC GAC GCA GTA ATT CCT AGC TCC TAT ATT GTT GTT ATG AAC GAT GAC GTT AGC ACC GCT GAG 144

$\begin{array}{llllllllllllllllllllllllll}49 & F & S & T & H & R & E & W & A & T & N & V & H & A & R & L & S & R & R & K & N & G & E & T & G\end{array}$ TTC AGC ACG CAT CGT GAA TGG GCT ACA AAT GTT CAT GCT CGC CTT TCT CGC CGT AAA AAT GGG GAG ACT GGA 216

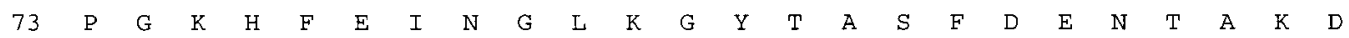
CCA GGA AAG CAT TTC GAA ATC AAT GGT CTG AAA GGA TAT ACT GCT AGC TTC GAC GAG AAT ACT GCT AAG GAT 288

\begin{tabular}{llllllllllllllllllllllllllll}
97 & $I$ & $A$ & $\mathbb{N}$ & $\mathrm{D}$ & $\mathrm{P}$ & $\mathrm{A}$ & $\mathrm{V}$ & $\mathrm{K}$ & $\mathrm{Y}$ & $\mathrm{I}$ & $\mathrm{E}$ & $\mathrm{P}$ & $\mathrm{D}$ & $\mathrm{M}$ & $\mathrm{I}$ & $\mathrm{V}$ & $\mathrm{N}$ & $\mathrm{A}$ & $\mathrm{T}$ & $\mathrm{A}$ & $\mathrm{N}$ & $\mathrm{V}$ & $\mathrm{V}$ & $\mathrm{Q}$ \\
\hline
\end{tabular} ATT GCC AAT GAC CCG GCG GTC AAG TAC ATC GAA CCA GAC ATG ATT GTG AAT GCA ACC GCG AAC GTG GTT CAA 360

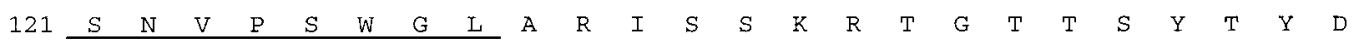
TCC AAT GTT CCT TCA TGG GGT CTT GCT CGC ATA TCT AGC AAg AGA ACT GGT ACC ACC AGC TAT ACA TAT GAT 432

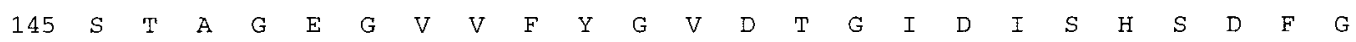
TCT ACT GCC GGT GAG GGC GTA GTA TTC TAC GGC GTC GAC ACC GGT ATT GAT ATC TCA CAC TCC GAC TTT GGG 504

$\begin{array}{llllllllllllllllllllllllllllll} & 169 & G & R & A & K & W & G & T & N & V & V & D & N & D & N & T & D & G & N & G & H & G & T & H & T\end{array}$ GGC CGT GCT AAA TGG GGT ACC AAC GTT GTT GAT AAT GAT AAT ACC GAC GGT AAT GGA CAT GGC ACT CAC ACT 576

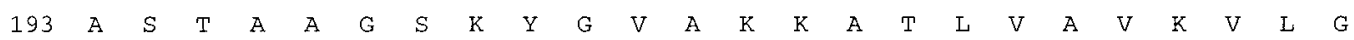
GCG TCT ACT GCG GCC GGT AGC AAG TAC GGT GTT GCA AAA AAG GCC ACT CTC GTC GCT GTC AAG GTC CTT GGT 648

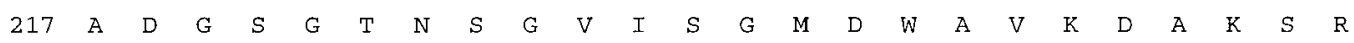
GCC GAT GGT TCT GGC ACT AAC TCA GGT GTC ATC TCT GGT ATG GAC TGG GCT GTT AAA GAT GCA AAG TCC CGC 720

$\begin{array}{llllllllllllllllllllllllllllllllll}241 & G & A & N & G & K & Y & V & M & N & T & S & L & G & G & E & F & S & K & A & V & N & D & A & A\end{array}$ GGC GCC AAC GGA AAG TAT GTT ATG AAT ACG TCT CTC GGC GGT GAG TTT TCC AAG GCT GTG AAT GAC GCC GCT 792

$\begin{array}{llllllllllllllllllllllllll}265 & A & N & V & V & K & S & G & I & F & L & S & V & A & A & G & N & E & A & E & N & A & S & N & S\end{array}$ GCC AAT GTT GTA AAG TCC GGT ATC TTC CTC TCC GTT GCT GCT GGT AAT GAG GCC GAG AAT GCG AGC AAC AGT 864

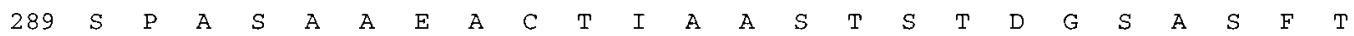
TCT CCT GCG TCT GCT GCG GAA GCA TGC ACT ATC GCG GCC TCT ACT AGC ACA GAT GGC AGC GCT TCA TTC ACG 936

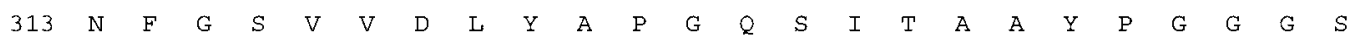
AAC TTC GGC TCC GTT GTT GAC CTC TAT GCT CCT GGG CAG AGT ATC ACG GCT GCA TAC CCT GGC GGT GGC TCG 1008

$\begin{array}{lllllllllllllllllllllllllllllllllll}337 & \mathrm{~K} & \mathrm{~T} & \mathrm{~L} & \mathrm{~S} & \mathrm{G} & \mathrm{T} & \mathrm{S} & \mathrm{M} & \mathrm{A} & \mathrm{A} & \mathrm{P} & \mathrm{H} & \mathrm{V} & \mathrm{A} & \mathrm{G} & \mathrm{V} & \mathrm{A} & \mathrm{A} & \mathrm{Y} & \mathrm{L} & \mathrm{M} & \mathrm{A} & \mathrm{L} & \mathrm{E}\end{array}$ AAG ACC CTA TCT GGA ACC TCC ATG GCC GCT CCA CAC GTT GCT GGT GTC GCC GCA TAT CTG ATG GCT CTC GAG 1080

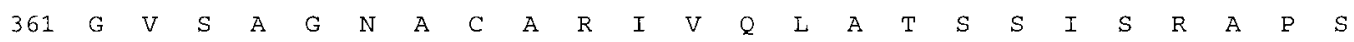
GGC GTG TCA GCT GGT AAC GCC TGT GCC CGT ATT GTC CAG CTC GCT ACC TCG TCA ATC TCT AGG GCT CCC TCT 1152

$\begin{array}{llllllllllllllllllllll}385 & G & T & T & S & K & \text { L } & \text { L } & Y & N & G & I & N & V\end{array}$

GGC ACT ACA AGC AAG CTT CTA TAC AAT GGC ATC AAC GTC TAA

1194

FIG. 2. Nucleotide and deduced amino acid sequences of Pen $n$ 13. The nucleotide sequence is shown bel ow the amino acid sequence. The numbers on the right of the figure indicate the positions of the nucleotide sequence. The numbers on the left of the figure indicate the position of the amino acid sequence. $\mathrm{N}$-terminal segment as determined by protein sequencing is underlined. The arrow indicates the start of the 90-amino-acid propeptide which is preceded by a 25-amino-acid signal peptide. Nucleotide sequence has been submitted to GenBank/E MBL/ DDJ B/Databases under the Accession No. AF 193420.

that the recombinant Pen $n 13$ exhibited the same antigenicity as native Pen c 1.

\section{Cross-Inhibition Assays of Pen $\mathrm{n} 13$}

For cross-inhibition assays, a serum pool of moldallergic patients was tested for IgE-binding to natural Pen c 1 (Fig. 4C). Preincubation of the serum pool with
rPen $n 13$ resulting in completely depletion of IgE binding to Pen $\mathrm{c} 1$, whereas preincubation of the serum pool with BSA did not influence the binding intensity and no reduction in the intensity of the allergen band was observed for the buffer control. The result indicated that the rPen $\mathrm{n} 13$ contains all the IgE epitopes of Pen c 1 allergen recognizable by the sera from pa- 


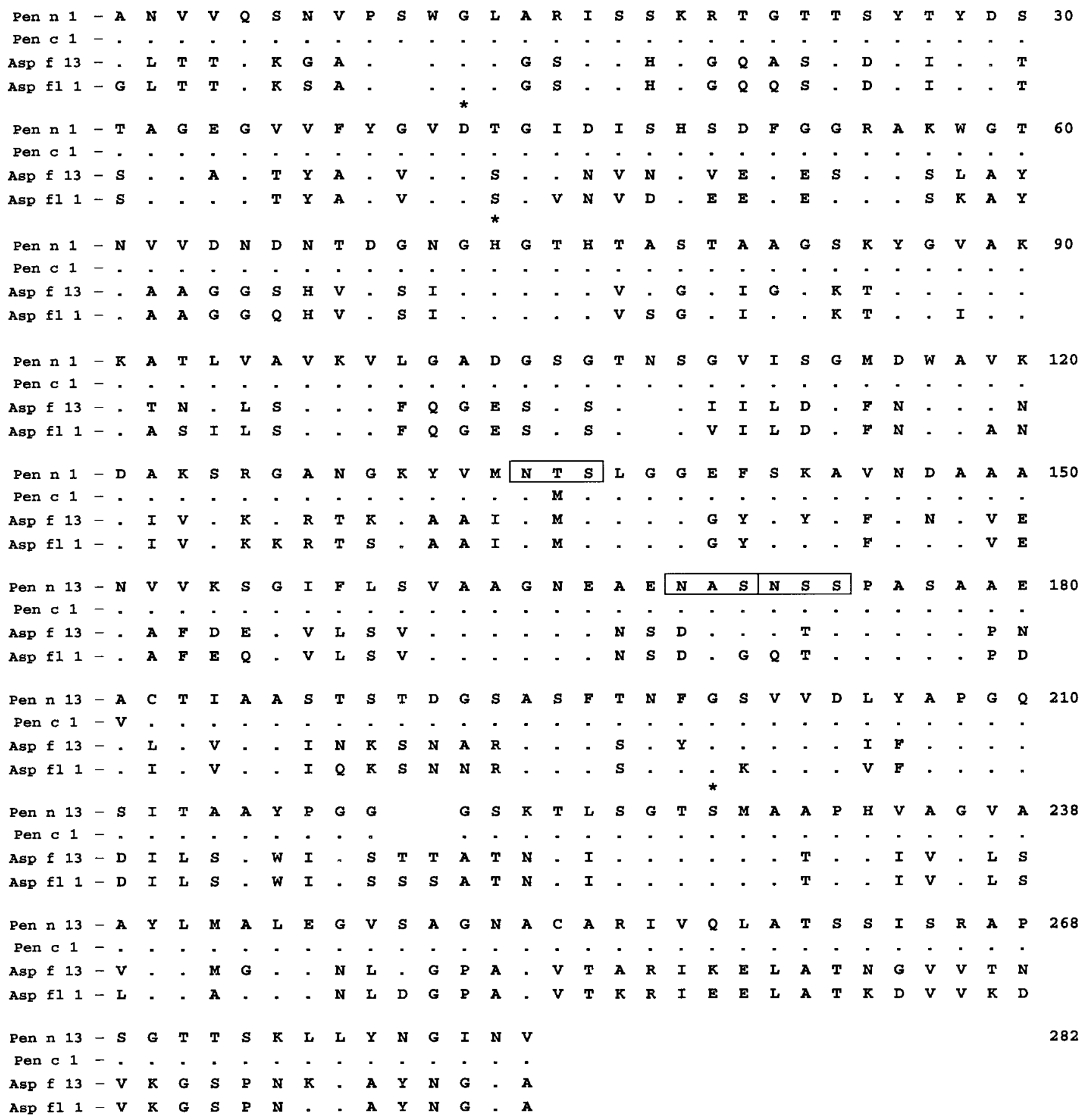

FIG. 3. Multiple sequence alignment of Pen $\mathrm{n} 13$ with other related mold allergens. The numbering system is based on the sequence of Pen $\mathrm{n} 13$ as reference. The gaps are introduced for optimal alignment and maximum homology among all compared sequences. Identical amino acids are indicated by dots. The highly conserved and consensus amino acid residues involved in the active site are indicated by asterisks. The potential consensus sequences for $\mathrm{N}$-glycosylation are boxed.

tients allergic to the Penicillium genus in general. Therefore judging by inhibition competition assays of Pen c 1 and rPen n 13, it is possible that similar or identical B-cell epitopes may exist between allergens of P. notatum and P. citrinum.

\section{Comparison of Antigenic Index of Different Mold Allergens}

For each allergen a plot made of the "antigenic index" against thethe polypeptide chain is shown in Fig. 
A

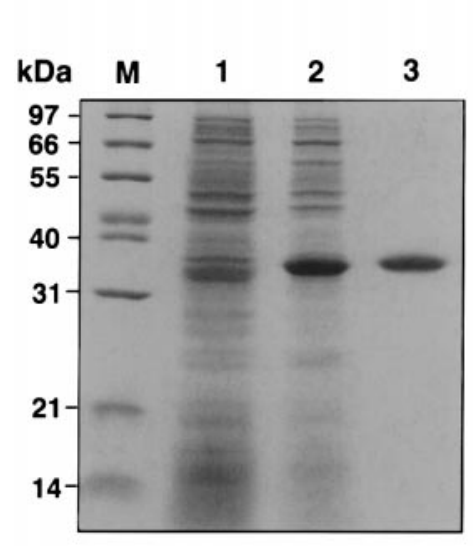

B

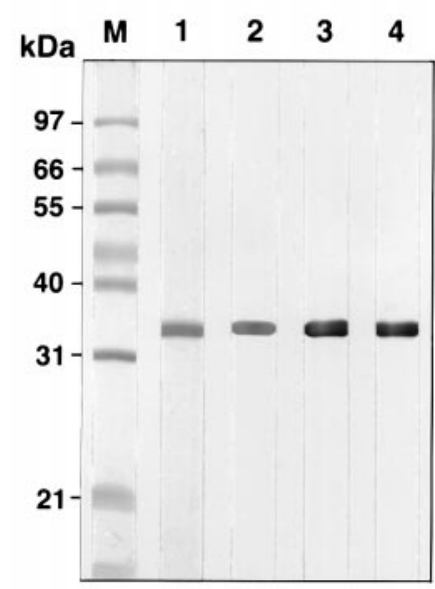

C

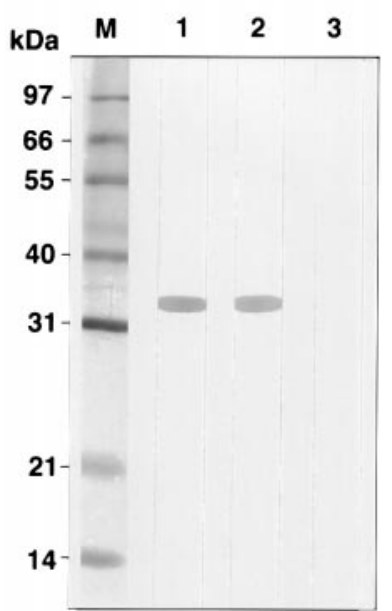

FIG. 4. Immunological characterization of recombinant Pen $n 13$ ( $r$ Pen $n$ 13). (A) Coomassie brilliant blue-stained SDS-PAGE for the expression and purification of rPen n 13. M, molecular mass markers; lane 1, non-IPTG-induced E. coli cell lysate; Iane 2, IPTG-induced rPen n 13 expression from $\mathrm{E}$. coli cell lysate; lane 3, rPen $\mathrm{n} 13$ purified after affinity chromatography. (B) I mmunodetection of rPen $\mathrm{n}$ 13. M, molecular mass markers; lane 1, purified rPen n 13 stained with Coomassie blue; lane 2, purified rPen n 13 detected by IgE of pool ed sera; lanes 3 and 4 , purified rPen $n 13$ detected by mAbs D7B8 and 55A, respectively. (C) Cross-reactivity inhibition experiments. Purified Pen c 1 was separated by SDS-PAGE and transferred onto PVDF membranes. Cross-reactivity inhibition was assayed by lane 1, preincubation of pooled sera with PBS buffer; lane 2, preincubation of pooled sera with BSA; lane 3, preincubation of pooled sera with rPen n 13; M, molecular mass markers.

5. These analyses reveal several potential antigenic sites, i.e. regions of epitopes recognized by existing antibodies. Antigenicity predictions indicated that there are ten epitopes of high antigenic indices in the Pen c 1 and Pen n 13 and about six such epitopes for Asp $f 13$ and Asp fl 1. It is of interest to find that there

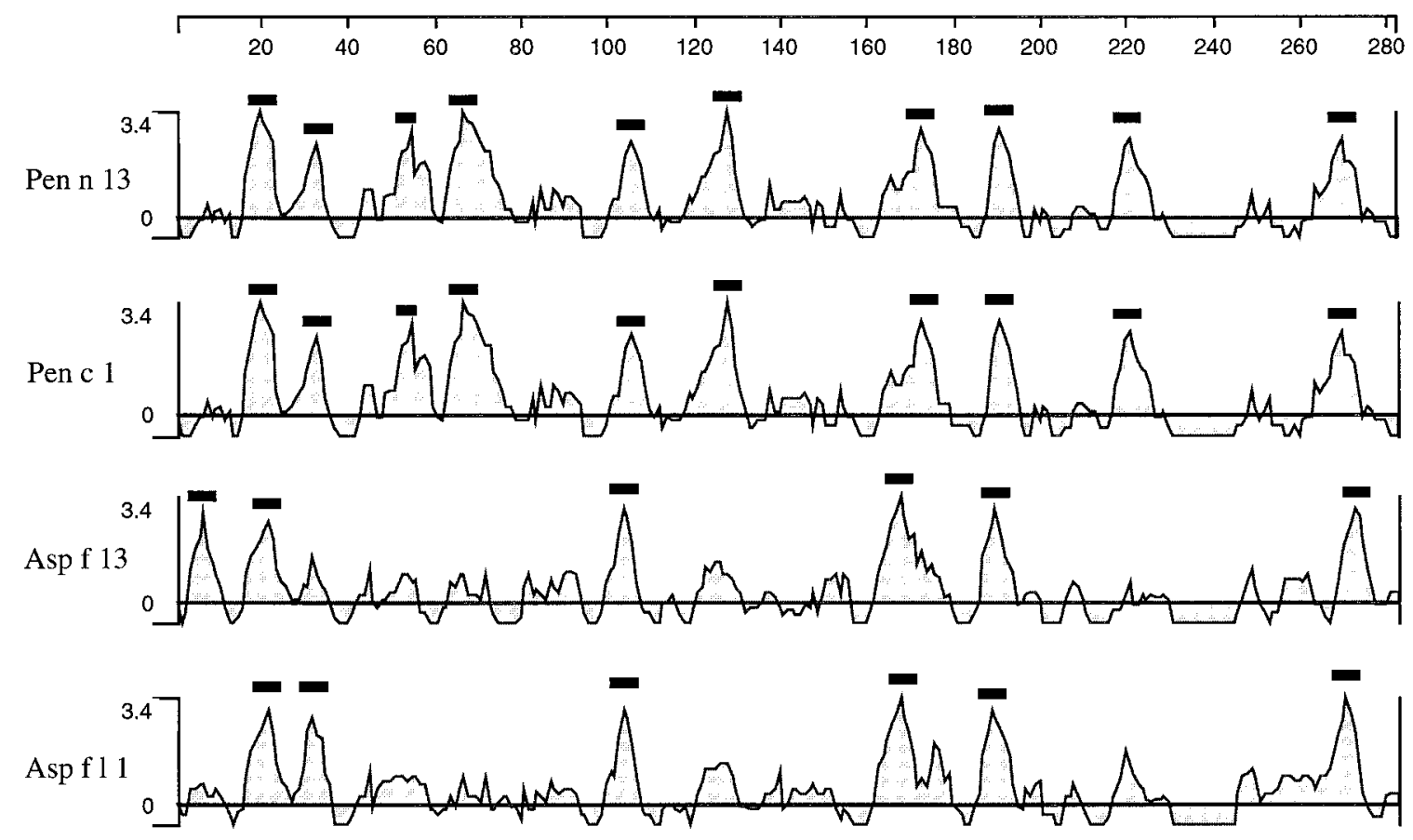

FIG. 5. Comparison of "Antigenic index" of different mold allergens. The deduced amino acid sequences of various mold allergens were analyzed by using the Lasergene software package (DNASTAR Inc.). The regions indicated by bars show the potential epitopes with high antigenic indices. 
are about five similar epitope regions present in these four mold allergens, suggesting that these allergens can show antigenic cross-reactivity.

\section{CONCLUSION}

In summary, the facile cloning approach presented here for the production of recombinant allergen and subsequent purification of the major mold allergen, Pen $n$ 13, appear to be successful. We demonstrated that the recombinant allergen of rPen $n 13$ exhibited a high potency of reactivity with IgE. The facile production and purification of recombinant mold allergens like rPen n 13 provide a straightforward strategy for a detailed study of epitope structure as well as the development of accurate diagnosis protocols leading to effective therapeutic strategies. The results also reveal the high similarity among alleregns from different mold species both on the protein structural levels and immunol ogical cross-reactivity. It also leads to the supposition that mold allergens of alkaline serine protease family represent major and prevalent aeroallergens.

\section{ACKNOWLEDGMENT}

This work was supported by the National Science Council (NSC Grant-89-2316-B-002-019 to L.-P. Chow) Taipei, Taiwan.

\section{REFERENCES}

1. Burge, H. A. (1985) Clin. Rev. Allergy 3, 319-329.

2. Horner, W. E., Helbling, A., Salvaggio, J. E., and Lehrer, S. B. (1995) Clin. Microbiol. Rev. 8, 161-179.
3. Su, N. Y., Yu, C. J ., Shen, H. D., Pan, F. M., and Chow, L. P. (1999) Eur. J . Biochem. 261, 115-123.

4. Chow, L. P., Su, N. Y., Yu, C. J ., Chiang, B. L., and Shen, H. D. (1999) Biochem. J. 341, 51-59.

5. Shen, H. D., Liaw, S. F., Lin, W. L., Ro, L. H., Yang, H. L., and Han, S. H. (1995) Clin. Exp. Allergy 25, 350-356.

6. Shen, H. D., Lin, W. L., Tsai, J. J ., Liaw, S. F., and Han, S. H. (1996) Clin. Exp. Allergy 26, 444-451.

7. Yu, C. J ., Chiou, S. H., Lai, W. Y., Chiang, B. L., and Chow, L. P. (1999) Biochem. Biophys. Res. Commun. 261, 669- 675.

8. Shen, H. D., Lin, W. L., Tam, M. F., Wang, S. R., Tzean, S. S., Huang, M. H., and Han, S. H. (1999) Clin. Exp. Allergy 29, $642-651$.

9. King, T. P., Hoffman, D., Lowenstein, H., Marsh, D. G., PlattsMills, T. A. E., and Thomas, W. (1994) Int. Arch. Allergy Immunol. 105, 224-233.

10. Shen, H. D., Lin, W. L., Liaw, S. F., Tam, M. F., and Han, S. H. (1997) Clin. Exp. Allergy 27, 79-86.

11. Chow, L. P., Fukaya, N., Miyatake, N., Horimoto, K., Sugiura, Y., Tabuchi, K., Ueno, Y., and Tsugita, A. (1995) J . Biomed. Sci. 2, 343-352.

12. Laemmli, U. K. (1970) Nature 227, 680- 685.

13. Chomczynski, P., and Sacchi, N. (1987) Anal. Biochem. 162, 156- 159.

14. Ikemura, H., Takagi, H., and Inouye, M. (1987) J . Biol. Chem. 262, 7859-7864.

15. Shinde, U., and I nouye, M. (1994) J . Biochem. 115, 629-636.

16. J aton-Ogay, K., Suter, M., Crameri, R., Falchetto, R., Fatih, A., and Monod, M. (1992) FEMS Microbiol. Lett. 92, 163-168.

17. Chow, L. P., Liu, S. L., Yu, C. J ., Liao, H. K., Tsai, J . J ., and Tang, T. K. (2000) Biochem. J ., in press.

18. Estell, D., Graycar, T. P., Miller, J . V., Powers, D. B., Burnier, J. P., and Wells, J. A. (1986) Science 233, 659-663. 